\title{
artigo
}

Fernandes, D. M. A. P., Morais, M. M. M., Santos, M. L. H., Sales, M. M., Sousa, V. C.

O uso do portfólio avaliativo em medicina: revisitando conceitos e experiências

\section{O uso do portfólio avaliativo em medicina: revisitando conceitos e experiências}

The use of the evaluative portfolio in medicine: revisiting concepts and experiences

El uso del portafolio evaluativo en medicina: revisitando conceptos y experiencias

\section{RESUMO}

Objetivo: o objetivo do presente estudo foi revisar como os portfólios são utilizados, uma vez que, pouco se sabe sobre a melhor forma de implementá-los na graduação, bem como fraquezas e potencialidades. Método: realizou-se uma revisão integrativa de artigos selecionados nas bases de dados da LILACS, MEDLINE e SciELO com os termos "portfólio" e "medicina" ligados pelo operador booleano "AND". Resultados: esta busca encontrou 55 artigos que após leitura de títulos e resumo foram excluídos 40. Conclusão: as referências elencadas reforçam que os portfólios são instrumentos que apoiam a educação médica baseada em competências desde que tenha objetivos claros e métodos eficazes de avaliação. São também indicados formatos digitais para estas ferramentas. Destarte, experiências locais com o uso de portfólios devem ser estimuladas.

DESCRITORES: Avaliação; Educação Médica; Avaliação Educacional.

\section{ABSTRACT}

Objective: The aim of this study was to review how portfolios are used since little is known about the best way to implement them at undergraduate level, weaknesses and strengths. Method: An integrative review of selected articles in the LILACS, MEDLINE e ScIELO databases was carried out with the terms "portfolio" and "medicine" with the Boolean operator "AND". Results: This search found 55 articles that after reading the titles and abstract were excluded 40. Conclusions: The listed references reinforce that portfolios are instruments that support competency-based medical education as long as it has clear objectives and effective assessment methods. Digital formats for these tools are also indicated. Therefore, local experiences with the use of portfolios should be encouraged.

DESCRIPTORS: Assessment; Medical Education; Educational Assessment.

\section{RESUMEN}

Objetivo: El objetivo de este estudio fue revisar cómo se utilizan los portafolios ya que se sabe poco sobre la mejor manera de implementarlos a nivel de pregrado, así como debilidades y fortalezas. Metodo: Se realizó una revisión integradora de los artículos seleccionados en la base de datos de las bases LILACS, MEDLINE e ScIELO con los términos "portafolio" y "medicina" con el operador booleano "Y". Resultados: Esta búsqueda encontró 55 artículos que luego de leer los títulos y el resumen fueron excluidos 40. Conclusiones: Las referencias enumeradas refuerzan que los portafolios son instrumentos que apoyan la educación médica basada en competencias siempre que tenga objetivos claros y métodos de evaluación efectivos. También se indican los formatos digitales para estas herramientas. Por lo tanto, deben fomentarse las experiencias locales con el uso de carteras.

DESCRIPTORES: Evaluación; Educación médica; Evaluación educativa.

RECEBIDO EM: 01/11/2021 APROVADO EM: 05/01/2022

\section{Denise Mota Araripe Pereira Fernandes}

Docente e preceptora da Residência em Medicina de Família e Comunidade (MFC) e do Internato em Atenção Primária à Saúde da Secretaria Municipal de Saúde de João Pessoa em parceria com a Faculdade de Ciências Médicas da Paraíba (FCM/PB Afya) ORCID: 0000-0001-7804-882X

\section{Melissa Maria Medeiros de Morais}

Residente em MFC pela SMS/JP.

ORCID: 0000-0003-2336-3907

Matheus Lucas Henriques Santos

Residente em MFC pela SMS/JP.

ORCID: 0000-0001-6342-9187 
Marília Moreira Sales

Residente em MFC pela SMS/JP.

ORCID: 0000-0002-5583-9458

Vinícius Carvalho Sousa

Residente em MFC pela SMS/JP.

ORCID: 0000-0002-6949-1640

\section{INTRODUÇÃO}

0 mundo contemporâneo é desafiador à formação de profissionais na área da saúde por demandar para além dos conhecimentos técnicos desconstruções conceituais e enfrentamento de alguns saberes consagrados como "hegemônicos" ainda que careçam de validação científica atual. Em face de tal realidade, as Diretrizes Curriculares Nacionais de Medicina de 2014 passaram a recomendar o desenvolvimento de competências a partir de experiências práticas. Tais mudanças se distanciam da obtenção mecânica de conhecimento e abrem espaço para uma educação médica mais atual, porém com inúmeros desafios ao ensino e avaliação adequados. 1

Muitos educadores acreditavam que "os diários de campo" ocupariam esta lacuna de dispositivos avaliativos, mas estes têm sido substituídos por portfólios, pela ênfase na reflexão crítica e capacidade de direcionar o aprendizado em processos cíclicos e autorregulados.2 O objetivo do portfólio é estimular a aprendizagem experiencial e melhorar a qualidade do cuidado. Ademais, novas formas de aprender invariavelmente requerem diferentes formatos de avaliar, com premissas mais abrangentes e dinâmicas, implicando reflexão crítica sobre as práticas vividas no sentido de visualizar avanços e resistências.3

Ao se considerar o componente formativo na medicina, o portfólio revela-se como um instrumento capaz de capturar tal complexidade, devido ao dinamismo e a abertura para o mundo, refletindo uma prática integrada. É um método de avaliação inovador, ao considerar os discentes como sujeitos ativos, destacando sua participação na seleção dos conteúdos procurados, proporcionando reflexão e criticismo.4 Destarte,

\section{Muitos educadores} acreditavam que

“os diários de

campo" ocupariam

esta lacuna de

dispositivos

avaliativos, mas

estes têm sido

substituídos por

portfólios, pela

ênfase na reflexão

crítica e capacidade

de direcionar o

aprendizado em

processos cíclicos e

autorregulados o portfólio entrecruza outros formatos de avaliação conseguindo refletir diversas vivências a exemplo do Mini Exame Clínico ou de casos clínicos que podem ter sido dramatizados através do Exame Clínico Objetivo Estruturado com pacientes-atores. 5

Enquanto ferramenta, pode arquivar trabalhos feitos pelos alunos com o objetivo de problematizar a realidade, vislumbrando o progresso e a qualidade dos registros, elencando-se as conquistas de ensino-aprendizagem em uma área específica ou interseccionada. É uma técnica que informa sobre as competências que o discente pode demonstrar, evidenciando as tarefas realizadas. São passíveis de autoavaliação, mas requerem guia de um professor que conheça o instrumento, compreendendo o protagonismo do discente e o desenvolvimento da escrita para reflexão e análise. Não se trata de uma nova forma de publicação de notas e catalogação de escritos, e sim da análise participativa no diálogo.6

Supõe-se, ainda, que portfólios contribuem para a aprendizagem autorregulada (AAR), na qual alunos são metacognitiva, motivacional e comportamentalmente participantes ativos. A literatura relata os seguintes processos de AAR apoiados por portfólios: autoavaliação, feedback, definição de metas, planejamento e monitoramento. A AAR é uma habilidade essencial para estudantes de medicina que trabalham e aprendem em locais comunitários-clínico-cirúrgicos que podem ser imprevisíveis. É estimado pelos pesquisadores, mas ambivalente para os alunos que relatam desde aprendizagem significativa a reflexões de valor limitado. Tais perdas podem ser explicadas por curto tempo para completá-lo, falta de propósito, foco no docente ao invés do discente em autodesenvolvimento.7

Nesse sentido, o objetivo do presente estudo é revisar de maneira integrativa como 
os portfólios são utilizados no campo da educação médica, uma vez que pouco se sabe sobre a melhor forma de implementar a aprendizagem baseada em portfólio na graduação médica. A questão de pesquisa é: o que define o portfólio avaliativo em fortalezas e fraquezas e como tem sido utilizado nas diferentes experiências nos processos de ensino-aprendizagem.

\section{MÉTODO}

Realizou-se uma revisão Integrativa da Literatura (RIL) de artigos selecionados nas bases de dados LILACS, MEDLINE e SciELO de outubro a novembro de 2021. Tal método foi escolhido por envolver a organização e a discussão de um assunto de pesquisa de forma verticalizada, lidando com diversos tipos de pesquisas tratando do mesmo tema. 8

Utilizou-se os seguintes descritores de busca: "Portfólio" e "Medicina" com o operador booleano "AND". Tal busca foi refinada em assuntos principais - "avaliação educacional", "educação médica" e "educação de graduação em medicina" - buscando publicações dos últimos 5 anos, nos idiomas inglês, português e espanhol. Foram encontrados 55 artigos, porém após leitura de títulos e resumos foram excluídos 25 artigos que não tratavam sobre portfólio dentro do contexto da avaliação médica, assim como também foram excluídos 9 artigos que usaram de portfólio para aquisição de competências de pós-graduação e não da graduação em medicina - mote da presente revisão, 4 artigos sobre portfólio em outros cursos da saúde que são a medicina e 2 artigos duplicados. Assim, os critérios de inclusão selecionaram 15 artigos que utilizaram os portfólios enquanto ferramenta de avaliação no curso de graduação em medicina.

\section{RESULTADOS}

Após a leitura de todos os resumos aplicou-se os critérios de exclusão. Foram selecionados 15 artigos segundo os seguintes critérios: abordagem do uso do portfólio durante a graduação em medicina, publicações dos últimos cinco anos e nos idiomas português, inglês e espanhol. Os artigos selecionados foram lidos na íntegra e sumarizados. Abaixo, foi organizado um quadro com os artigos elencados e um resumo dos principais achados.

\section{DISCUSSÃO}

O portfólio para fins de avaliação é uma resposta às mudanças na educação médica, incluindo a ênfase no profissionalismo e na necessidade de dar aos alunos mais responsabilidade. Este formato de avaliação é utilizado durante todos os períodos da gra-

Quadro 1. Artigos selecionados pela RIL.

$\begin{array}{ccc}\begin{array}{c}\text { Base de } \\ \text { dados }\end{array} & \text { Título } & \begin{array}{l}\text { Tipo de } \\ \text { estudo }\end{array}\end{array}$

Principais Achados

Portafolio de evaluación: una experiencia

1. LILACS novedosa en la asignatura Introducción a la Medicina General Integral
Rodriguez Cardenas et al. EDUMECEN-

Relato de ex- TRO vol.12 no.4 Santa periência Clara out.-dez. 2020 Epub 30 de dezembro de 2020
Aplicação do portfólio do curso de Introdução à Medicina Geral Integral no primeiro ano do curso para avaliação de atividades educativas no trabalho. 0 portfólio representa um novo cenário de avaliação, é uma técnica que informa sobre as competências que o aluno pode demonstrar, bem como a natureza e utilização do processo de aprendizagem, permite acumular evidências e inclui a avaliação dos aspectos educacionais num elo indissociável e instrutivo.
How is self-regulated learning documen-

2. MEDLINE ted in e-portfolios of trainees? A content analysis.
Análise descritiva de banco de dados VAN DER GULDEN at composta por inúmeros processos de reflexão e al. BMC Med Educ; feedback, estabelecimento de metas, planejamen20(1): 205, 2020 Jun to e monitoramento. 0 conteúdo do e-portfólio 26. mostrou documentação limitada de processos de do do portfólio eletrônico de médicos estagiários

Visão sobre a documentação de processos de aprendizagem autorregulada, dentro do conteúaprendizagem autorregulada, mas tal documentação requer tempo e exige um alto nível de introspecção e habilidades de redação. 
Portafolio en pregrado de Medicina: im-

3.

LILACS pacto educacional a
10 años de su imple-
mentación

Use of an e-portfolio mapping tool: connecting experiences,

4. MEDLINE analysis and action by learners. Perspectives on Medical Education.

Evaluation of a portfolio-based course

5. MEDLINE on self-development for pre-medical students in Korea

A heterogeneidade na correção dos portfólios no curso de 6. SciELO Medicina: percepção discente. Interface Comunicação, Saúde, Educação.

Reyes Diego, Isbej O portfólio inclui casos clínicos, medicina narrativa, Lorena, Uribe Javier, cuidados paliativos e medicina baseada em evidênRuz Cristián, Pizar- cias. A percepção geral do aluno foi positiva, desro Margarita, Walker tacando-se o desenvolvimento da análise crítica, Rosa et al. Rev. méd. raciocínio clínico e profissionalismo. A entrega de Chile [Internet]. 2019 feedback e avaliação de aprendizagem, permitiu Jun [citado 2021 que os alunos obtivessem notas excelentes. Houve Out31 ];147(6):790 - apenas dois casos de plágio relatados. Foram publi798. cados 15 artigos e dois livros com 52 ensaios.

Demonstrar como essa ferramenta poderia ser usada para apoiar atividades de prática reflexiva. Heeneman, Sylvia Seguindo a abordagem gradual típica para o ciclo Driessen, Erik; Dur- reflexivo, dois tipos de mapas foram projetados, um mapa de gatilho e um mapa de competências.

Pesquisa me- ning, Steven J; Torre, todológica Dario. Perspect Med Educ ; 8(3): 197-200, 201906. Em um mapa de gatilho, o aluno reflete sobre uma aprendizagem concreta ou experiência de feedback. Em um mapa de competências, o aluno interpreta e sintetiza vários mapas de gatilho anteriores que, em última análise, levam à formulação de novos objetivos de aprendizagem.

Curso baseado em portfólio com o objetivo de fortalecer as capacidades dos alunos de medicina para

Pesquisa metodológica Rao, Dong Mi; Cho, A Eval Health Prof ; 16: 38, 2019.

Estudo descritivo, com abordagem qualitativa de análise dos dados
Nery NLM, Baracat ECE, e Bicudo AM. Inção, Saúde, Educação [online]. 2019, v. 23 [Acessado 310utubro 2021], e170348. terface - Comunica- a autogestão e o autoaperfeiçoamento. Os sujeitos deste estudo foram 97 alunos de um curso pré-médico. A experiência de aprendizagem foi a avaliação do professor sobre os portfólios e o programa fo avaliado com base nas respostas de 68 alunos a uma pesquisa de 32 itens.

Na Unicamp, o portfólio foi implementado na Medicina em 2001, - é desenvolvido no quarto ano, quando os discentes atuam nas Unidades Básicas de Saúde. Cada portfólio é corrigido por um docente, e, após as considerações necessárias de cada tópico, atribui-se uma nota. 0 objetivo deste estudo foi compreender a percepção dos discentes sobre a correção do portfólio pelo grupo docentes. Os alunos consideraram que a avaliação por meio do portfólio necessita ser aprimorada com definição clara dos critérios de correção. Na percepção discente, destaca-se a heterogeneidade e a subjetividade dos docentes nessa correção. 


\section{artigo}

Fernandes, D. M. A. P., Morais, M. M. M., Santos, M. L. H., Sales, M. M., Sousa, V. C

O uso do portfólio avaliativo em medicina: revisitando conceitos e experiências

Assessing learning in

7. MEDLINE the adaptive curriculum. Medical Teacher.
Patricia A. Carney, George C. Mejicano, TraPesquisa metodológica

cy Bumsted \& Mark

Quirk (2018): DOI:

2018.1484083

Students' perception of portfolio as a
10.1080/0142159X.

8. MEDLINE learning tool at King

Avaliações adaptativas devem medir a capacidade do aluno de observar mudanças na prestação de cuidados e como implementá-las. Equilibrar avaliações formativas e somativas promoverá o aprendizado reflexivo para que cada aluno alcance seu potencial máximo. Descrevemos como duas escolas médicas estão abordando a avaliação adaptativa, incluindo o uso de sistemas de portfólio que abrangem experiências de ensino e aprendizagem, oferecendo rastreamento longitudinal em tempo real de dados digitais para melhorar a aprendizagem.

Diferença de foco entre os anos básicos e clínicos nos anos básicos, o foco dos alunos estava na aquisição de habilidades práticas, mas nos anos clínicos Fida, Nadia M., et al. eles se concentraram mais na aquisição de habilidaAbdulaziz University Medical School. Medical Teacher

Pesquisa qualitativa Medical Teacher, 1-10 doi:10.1080/014215 $9 \times .2018 .1466054$

Chae, Su Jin; Lee, Yong

Exploring the stratebuilding e-portfolios in medical schools todológica

Won. Korean J Med Educ ; 33(2): 133-137, 2021 Jun.
9. MEDLINE gies for successfully

Pesquisa medes complexas, incluindo a identificação e gerenciamento de problemas. No entanto, as respostas ao questionário revelaram uma tendência positiva na aceitação (crença no valor educacional) de portfólios entre os alunos e seus mentores, ao longo dos anos do programa.

Os alunos perceberam que os e-portfolios têm um alto grau de vantagem relativa, experimentabilidade e complexidade como características de resistência à inovação. Em relação ao risco percebido, eles não queriam que outras pessoas vissem suas informações, mas tinham um alto grau de demanda de comunicação com seus professores. 0 uso bem-sucedido de portfólios pode servir como uma ferramenta para gerenciamento e avaliação do aluno que pode refletir sua introspecção, desenvolvimento pessoal e desempenho acadêmico.

Longitudinal evaluation of a pilot e-portfolio basesupervi-

10. MEDLINE sion programme for final year medical students: views of students, supervisors and new graduates
Pesquisa qualitativa
Vance, Gillian HS; Burford, Bryan; Shapiro, Ethan; Price, Richard. BMC Med Educ ; 17(1):

141, 2017 Aug 22.
Avaliamos a introdução de um piloto de supervisão baseado em e-portfólio formativo para estudantes de medicina do último ano, buscando opiniões de alunos, supervisores e graduados sobre o uso e os efeitos educacionais. Os alunos do último ano têm atitudes negativas em relação a um e-portfólio formativo, embora os benefícios para facilitar a transição educacional sejam reconhecidos pelos graduados. Medidas para otimizar o tempo podem encorajar o uso. 


\begin{tabular}{|c|c|c|c|c|c|}
\hline 11 & SCIELO & \begin{tabular}{l} 
Portfólio Reflexivo: \\
subsídios filosóficos \\
para uma práxis nar- \\
rativa no ensino mé- \\
\multicolumn{1}{c}{ dico }
\end{tabular} & $\begin{array}{l}\text { Pesquisa qua- } \\
\text { litativa }\end{array}$ & $\begin{array}{c}\text { Stelet, Bruno Pereira } \\
\text { et al. Interface - Comu- } \\
\text { nicação, Saúde, Edu- } \\
\text { cação [online] 2017, v. } \\
21, \text { n. } 60 \text { [Acessado } 1 \\
\text { Novembro 2021] , pp. } \\
165-176\end{array}$ & $\begin{array}{l}\text { Analisar o Portfólio Reflexivo como dispositivo pe- } \\
\text { dagógico para o exercício da narratividade na me- } \\
\text { dicina. Ao produzir uma análise teórica acerca do } \\
\text { porttólio, construímos um diálogo epistemológico } \\
\text { entre Saúde Coletiva, Educação e Filosofia, no sen- } \\
\text { tido de oferecer elementos para se pensar o exercí- } \\
\text { cio de tecnologias relacionais fundamentais para o } \\
\text { cuidado em saúde. }\end{array}$ \\
\hline 12. & MEDLINE & $\begin{array}{l}\text { Evaluation of the free, } \\
\text { open source software } \\
\text { WordPress as elec- } \\
\text { tronic portfolio sys- } \\
\text { tem in undergraduate } \\
\text { medical education. }\end{array}$ & $\begin{array}{l}\text { Pesquisa me- } \\
\text { todológica }\end{array}$ & $\begin{array}{l}\text { Avila, J., Sostmann, K., } \\
\text { Breckwoldt, J. et al.. } \\
\text { BMC Med Educ 16, } \\
157 \text { (2016). https:// } \\
\text { doi.org/10.1186/ } \\
\text { s12909-016-0678-1 }\end{array}$ & $\begin{array}{l}0 \text { ambiente do ePortfolio do WordPress permi- } \\
\text { tiu aos alunos um amplo espectro de atividades - } \\
\text { muitas vezes documentadas por celulares - como } \\
\text { coleta de evidências de multimídia, postagem de } \\
\text { reflexões, mensagens, publicação na web, apren- } \\
\text { dizagem colaborativa, gerenciamento de conheci- } \\
\text { mento em um sistema de gerenciamento de con- } \\
\text { teúdo. }\end{array}$ \\
\hline 13. & MEDLINE & $\begin{array}{l}\text { Feasibility and Ou- } \\
\text { tcomes of Imple- } \\
\text { menting a Portfolio } \\
\text { Assessment System } \\
\text { Alongside a Traditio- } \\
\text { nal Grading System. } \\
\text { Academic Medicine. }\end{array}$ & $\begin{array}{l}\text { Pesquisa me- } \\
\text { todológica }\end{array}$ & $\begin{array}{l}\text { O'Brien, Celia Laird; } \\
\text { Sanguino, Sandra M; } \\
\text { Thomas, John X; Gre- } \\
\text { en, Marianne M. Acad } \\
\text { Med ; 91(11): 1554- } \\
\text { 1560, 201611. }\end{array}$ & $\begin{array}{l}\text { Desenvolvimento e a implementação de um siste- } \\
\text { ma de portfólio eletrônico baseado em competên- } \\
\text { cias longitudinais ao lado de um currículo graduado } \\
\text { em uma escola de medicina dos EUA. Em } 2009, \\
\text { os autores desenvolveram um sistema de portfó- } \\
\text { lio que serviu como um repositório para todas as } \\
\text { avaliaçỗes dos alunos organizadas por domínio de } \\
\text { competência. Cinco competências foram selecio- } \\
\text { nadas para uma revisão sumativa do portfólio de } \\
\text { pré-curso. Os alunos enviaram reflexões sobre seu } \\
\text { desempenho. }\end{array}$ \\
\hline 14. & MEDLINE & $\begin{array}{l}\text { Content validity of } \\
\text { workplace-based } \\
\text { portfolios: A multi- } \\
\text {-centre study }\end{array}$ & $\begin{array}{l}\text { Estudo quali- } \\
\text { tativo multi- } \\
\text { cêntrico }\end{array}$ & $\begin{array}{l}\text { Michels, Nele R M., et } \\
\text { al. Med Teach; 38(9): } \\
\text { 936-45, } 2016 \text { Sep. }\end{array}$ & $\begin{array}{l}\text { Os portfólios são usados como ferramentas para } \\
\text { treinar e avaliar os alunos no internato por estu- } \\
\text { dantes de medicina durante o estágio. Para validar } \\
\text { seu conteúdo, desenvolvemos um inventário. Dois } \\
\text { avaliadores avaliaram cada portfólio e indicaram } \\
\text { para cada item se o portfólio fornecia informações } \\
\text { suficientes para permitir uma avaliação satisfatória } \\
\text { do item. Este estudo dá mais crédito à evidência de } \\
\text { que os portfólios podem de fato avaliar adequada- } \\
\text { mente durante o internato. }\end{array}$ \\
\hline
\end{tabular}




\title{
artigo
}

Fernandes, D. M. A. P., Morais, M. M. M., Santos, M. L. H., Sales, M. M., Sousa, V. C.

O uso do portfólio avaliativo em medicina: revisitando conceitos e experiências

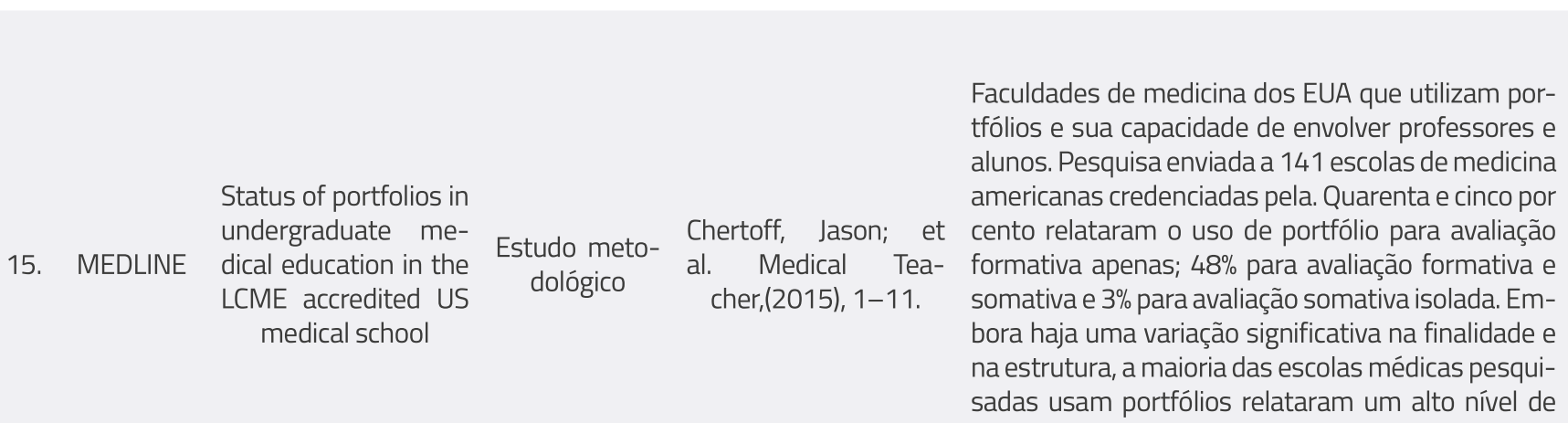
envolvimento com alunos e professores.
\end{abstract}

Fonte: elaborado pelos autores (2021).

duação porque fornece meios para avaliar a autoaprendizagem.3 Neste ínterim, os artigos 1, 2, 3 e 4 descrevem os novos cenários formativos e dialogam com a responsabilidade docente de motivar os alunos a construírem elementos significativos-simbólicos de aprendizagem. 5,6,7,8,9,10,11

No que tange à percepção discente, o artigo 2 reporta uma visão ambivalente dos alunos acerca da ferramenta, mas esta visão é não sustentada pelo artigo 3 , estudo de maior robustez por ser uma coorte de dez anos que acompanha a implementação dos portfólios. Já o quarto artigo demonstra e descreve que a percepção ambivalente dos alunos sobre o portfólio potencializou o design de uma ferramenta online capaz de ajudar construções mais estruturadas. Embora as escolas estejam desenvolvendo sistemas modernos e incentivando os alunos a usá-los, os artigos 5, 6, 7, 8 e 9 descrevem que os estudantes de medicina parecem insatisfeitos e sugere que é necessário considerar as reclamações dos alunos, a fim de superar os motivos de sua resistência.12,13,14,15

$\mathrm{O}$ artigo 10 também traz queixas discentes, apontando a atividade como uma "carga" não alinhada com os processos de aprendizagem. Para alcançar melhores resultados, gastar tempo explicando e checando a compreensão dos conceitos subjacentes a um portfólio foi uma estratégia usada pelo grupo do quinto artigo da presente revisão. É uma ferramenta que contribui para a avaliação das atitudes e profissionalismo do aluno, fornecendo amostras padrão e

\section{Embora as}

escolas estejam

\section{desenvolvendo}

sistemas modernos

e incentivando os

alunos a usá-los,

os artigos 5, 6, 7,8

e 9 descrevem que

os estudantes de

medicina parecem

insatisfeitos e sugere

que é necessário

considerar as

reclamações dos

alunos, a fim de

superar os motivos

de sua resistência possibilitando oficinas de treinamento com feedback.10 Uma outra possibilidade é reservar mais tempo para a criação do portfólio ou até mesmo considerar uma oficina para elaborá-lo dentro das classes regulares em grupos pequenos ou individuais, minorando a carga de atividades pós-classe dos alunos.

Apesar da necessidade de ajustes, o portfólio apresenta diversas potencialidades: auxiliam o examinador a fazer uma interpretação abrangente e confiável do desempenho do educando de forma personalizada. Tais exemplos remetem ao currículo centrado no discente ao passo em que os responsabiliza por seu próprio aprendizado. $\mathrm{O}$ artigo 6 descreve a experiência do portfólio na Universidade de Campinas, em que o portfólio é utilizado no quarto ano do curso de medicina, no módulo "Atenção Integral à Saúde", quando o aluno atua nas UBS. As competências a serem desenvolvidas neste estágio compreendem o desenvolvimento do raciocínio clínico, do processo de cuidado, da relação médico-paciente, da responsabilização e da vivência no ambiente extra-hospitalar.12,22

Possíveis disparidades na correção dos avaliadores podem surgir, assim é necessário entender as etapas para implementação dos portfólios. Para minorar tais incongruências a implantação do portfólio enquanto ferramenta avaliativa prescinde de clara definição do propósito e do conteúdo desde que seja mantido espaço para o aluno descrever os encontros com os pacientes, o 
checklist de autoavaliação, competências a serem avaliadas com sistema de marcação para cada um dos resultados de aprendizagem, examinadores treinados e planejamento do processo de implementação com cronograma, orientação dos alunos e diretrizes para decisões - caso sejam usados para aprovação/reprovação, assim os padrões precisam ser especificados para que não haja dúvidas. 10,12

Nesse sentido, o portfólio versa preponderantemente sobre conteúdo e a articulação de sistemas digitais de armazenamento pode gerar estruturas de dados potentes aos grupos educacionais nos quais várias escolas médicas colaboram. $\mathrm{O}$ artigo 7 examina as características de um currículo adaptável e o uso de diferentes tipos de avaliações do aluno e avaliação do programa em duas escolas de medicina, descrevendo o justo desenvolvimento de sistemas de portfólio para fornecer acesso a dados e como eles podem ser usados para melhorar continuamente os programas educacionais e os resultados acadêmicos individuais. Incorporam avaliações frequentes, acompanhamento da progressão através do portfólio eletrônico e personalização de elementos curriculares para cada universitário.13

O sistema de portfólio eletrônico para rastreamento de alunos gera, assim, dados de pesquisa e avaliação para melhoria educacional. Normalmente, são implementados com softwares desenvolvidos internamente sob medida para as necessidades da instituição. $\mathrm{O}$ artigo 12 tensiona o custo e as dificuldades de ajustar aos requisitos específicos da instituição, assim sugerem softwares livres que consumam menos recursos, permitindo ajustes e que já tenham sido implementados com sucesso para a construção de plataformas de baixo custo para atividades de e-learning.14

$\mathrm{O}$ artigo 13 narrou a experiência de customizar a plataforma para controlar de forma personalizada as competências que o grupo colegiado pretendeu potencializar nos alunos e relatam que tal construção possibilitou o entendimento e o desempenho deles em competências orientadas para o comportamento de uma forma que não era possível antes de seu desenvolvimento. 15
Apenas o artigo 14 discute o uso da ferramenta no internato e relata que esta é válida, desde que o conteúdo tenha obje-

\section{Os portfólios}

\section{são ferramentas}

potentes para

a aquisição do

profissionalismo

em medicina,

devendo ter o uso

encorajado pelos

grupos educacionais

preocupados com o

engajamento social

dos estudantes.

Ainda existem

muitas lacunas na

literatura e espaços

para pesquisas

longitudinais sobre

o tema tivos claramente determinados e selecione de forma apropriada instrumentos de avaliação adequados às competências pretendidas. 16

Revisitando termos conceituais do portfólio enquanto dispositivo, os autores do artigo 11 produzem um ensaio rico em contribuições da Filosofia capaz de produzir nexos conceituais para defender o portfólio como dispositivo pedagógico potente à produção narrativa na prática médica, norteando metodologias pedagógicas.17,18 Permite o desenvolvimento de valores éticos e consciência social dos aprendizes ao se valer do encontro com o paciente para justificar tal atividade. Ademais, recruta dimensões cognitivas e comunicacionais, produzindo conhecimento por meio deste reconhecimento. $\mathrm{O}$ artigo 15 descreve como boa parte das escolas americanas têm utilizado os portfólios em alguma medida nas suas práticas. 19,20

A presente revisão dialoga com a RIL feita por Garcia e Nascimento que mesclou artigos e projetos políticos pedagógicos, demonstrando que a aplicação do portfólio, sobressaem dificuldades relativas à manutenção do modelo biomédico e a falta de motivação para que os docentes se capacitem com apoio institucional. 21

\section{CONCLUSÃO}

Os portfólios são ferramentas potentes para a aquisição do profissionalismo em medicina, devendo ter o uso encorajado pelos grupos educacionais preocupados com o engajamento social dos estudantes. Ain$\mathrm{da}$ existem muitas lacunas na literatura $\mathrm{e}$ espaços para pesquisas longitudinais sobre o tema. A presente revisão integrou aspectos aplicativos do método e sugeriu, a partir das experiências coletadas, caminhos para potencializá-lo. Os dispositivos eletrônicos personalizados podem ser um caminho, porém os custos via de regra são impeditivos. Destarte, mais experiências de criação e aplicabilidade de portfólios integrando discentes e docentes podem enriquecer o campo da educação médica à medida em que mobilizam o uso da ferramenta. 


\section{REFERÊNCIAS}

1. Brasil. Ministério da Educação. Conselho Nacional de Educação. Câmara de Educação Superior. Resolução CNE/CES n 3 de 20 de junho de 2014. Institui diretrizes curriculares nacionais do curso de graduação em Medicina e dá outras providências. Diário Oficial da União. Brasília, 23 Jun. 2014; Seção 1, pp.8-11.

2. Fida NM, Hassanien M, Shamim MS, Alafari $R$, Zaini $R$, Mufti $S$, et al. Students' perception of portfolio as a learning tool at King Abdulaziz University Medical School. Medical Teacher. 2018 Jun 17;40(sup1):S104-13.

3. Marin MJS, Moreno TB, Moravcik MY, Higa E de FR, Druzian S, Francischetti I, et al. $\mathrm{O}$ uso do portfólio reflexivo no curso de medicina: percepção dos estudantes. Revista Brasileira de Educação Médica. 2010 Jun; 34(2):191-8.

4. Yoo DM, Cho AR, Kim S. Evaluation of a portfolio-based course on self-development for pre-medical students in Korea. Journal of Educational Evaluation for Health Professions. 2019 Dec 11;16:38.

5. Reyes D, Isbej L, Uribe J, Ruz C, Pizarro M, Walker R, et al. Portafolio en pregrado de Medicina: impacto educacional a 10 años de su implementación. Revista médica de Chile. 2019 Jun;147(6):790-8.

6. Rodríguez Cardenas M, Paz Paz O, Sanchez Perez II, Pérez Rodriguez LA, Rodríguez Cardenas M, Paz Paz O, et al. Portafolio de evaluación: una experiencia novedosa en la asignatura Introducción a la Medicina General Integral. EDUMECENTRO [Internet]. 2020 Dec 1 [cited 2021 Nov 1];12(4):235-41. Available from: http://scielo.sld.cu/scielo.php?script=sci_arttext\&pid=S2077-28742020000400235\&1$\mathrm{ng}=\mathrm{es}$.

7. van der Gulden R, Heeneman S, Kramer AWM, Laan RFJM, Scherpbier-de Haan ND, Thoonen BPA. How is self-regulated learning documented in e-portfolios of trainees? A content analysis. BMC Medical Education. 2020 Jun 26;20(1)

8. Botelho LLR, Cunha CCA, Macedo M. O método da revisão integrativa nos estudos organizacionais. Gestão e Sociedade. 2011;5(11):121-36.

9. Castanelli DJ, Weller JM, Molloy E, Bearman M. Shadow systems in assessment: how supervisors make progress decisions in practice. Advances in Health Sciences Education. 2019 Sep 3;25(1):131-47.

10. Harden R, Laidlaw J. Essential skills for a medical teacher: an introduction to teaching and learning in medicine. SECOND. Elsevier: 2017.

11. Vance GHS, Burford B, Shapiro E, Price R. Longitudinal evaluation of a pilot e-portfolio-based supervision programme for final year medical students: views of students, supervisors and new graduates. BMC Medical Education. 2017 Aug 22;17(1).

12. Chae SJ, Lee YW. Exploring the strategies for successfully building e-portfolios in medical schools. Korean Journal of Medical Education [Internet]. 2021 Jun 1 [cited 2021 Nov 1];33(2):133-7. Available from: https://pubmed.ncbi.nlm.nih.gov/33957730/

13. Heeneman S, Driessen E, Durning SJ, Torre D. Use of an e-portfolio mapping tool: connecting experiences, analysis and action by learners. Perspectives on Medical Education. 2019 May 16;8(3):197-200.

14. Nery N de ML, Baracat ECE, Bicudo AM. A heterogeneidade na correção dos portfólios no curso de Medicina: percepção discente. Interface - Comunicação, Saúde, Educação. 2019;23.

15. Carney PA, Mejicano GC, Bumsted T, Quirk M. Assessing learning in the adaptive curriculum. Medical Teacher. 2018 Aug 3;40(8):813-

16. Avila J, Sostmann K, Breckwoldt J, Peters H. Evaluation of the free, open source software WordPress as electronic portfolio system in undergraduate medical education. BMC Medical Education [Internet]. 2016 Jun 3 [cited 2021 Feb 26];16. Available from: https:// www.ncbi.nlm.nih.gov/pmc/articles/PMC4891874/

17. O'Brien CL, Sanguino SM, Thomas JX, Green MM. Feasibility and Outcomes of Implementing a Portfolio Assessment System Alongside a Traditional Grading System. Academic Medicine. 2016 Nov;91(11):1554-60.

18. Michels NRM, Avonts M, Peeraer G, Ulenaers K, Van Gaal LF, Bossaert LL, et al. Content validity of workplace-based portfolios: A multi-centre study. Medical Teacher. 2016 Jan 30;38(9):936-45.

19. Stelet BP, Romano VF, Carrijo APB, Teixeira Junior JE. Portfólio Reflexivo: subsídios filosóficos para uma práxis narrativa no ensino médico. Interface - Comunicação, Saúde, Educação [Internet]. 2016 Oct 24 [cited 2021 Mar 19];21(60):165-76. Available from: https:/www.scielo.br/pdf/icse/2016nahead/1807-5762icse-1807-576220150959.pdf

20. Chertoff J, Wright A, Novak M, Fantone J, Fleming A, Ahmed T, et al. Status of portfolios in undergraduate medical education in the LCME accredited US medical school. Medical Teacher. 2015 Dec 10;38(9):886-96

21. Garcia MAA, Nascimento GEA do. Aplicação do Portfólio nas Escolas Médicas: Estudo de Revisão. Revista Brasileira de Educação Médica [Internet]. 2019 Mar [cited 2021 Sep 6];43(1):163-74. Available from: https://www.scielo.br/j/rbem/a/b6Nx4BJ7n5T9fP6scbKDhqf/?format=pdf\&lang=pt.

22. Borges de Assis LM, Lima MP, Medeiros MMM, Fernandes MAP, Costa VFM, Falcão MM. (2021). Cuidado dentro de casa: reflexões sobre visitas domiciliares na Estratégia Saúde da Famíllia. Saúde Coletiva (Barueri), 11(62), 5072-5081. https://doi.org/10.36489/ saudecoletiva.2021v11i62p5072-5081. 